\title{
Study on the mechanical and morphological properties of toughened polypropylene blends for automobile bumpers
}

\author{
GuangYong Liu • GuiXue Qiu
}

Received: 3 June 2012/Revised: 7 August 2012/ Accepted: 6 November 2012/

Published online: 15 November 2012

(C) The Author(s) 2012. This article is published with open access at Springerlink.com

\begin{abstract}
Studies on the use of polyolefin elastomer (POE) and high density polyethylene (HDPE) for toughening polypropylene (PP) to meet the demands of automobile bumpers were conducted. The effect of the basic resin, POE and the influences of the POE amount and HDPE doses on the mechanical properties of the blended composites were discussed. The morphology of impact fracture sections were characterized by scanning electron microscopy (SEM) while the crystalline properties were investigated by DSC. The effect of the composites' morphology on mechanical properties was also discussed. Results showed that POE could improve the impact strength of PP while the use of HDPE had obvious effects on synergistic toughening. SEM images and DSC data analysis testified to the relationship between morphology, crystallinity and the mechanical properties.
\end{abstract}

Keywords Polypropylene - Polyolefin elastomer - Automobile bumper . Toughening modification

\section{Introduction}

Polypropylene (PP) is a thermoplastic with a number of desirable properties that make it a versatile material. However, the poor impact property, especially at low temperature, limits some of its applications. To achieve better properties, impact modifiers have been added to PP. Among the impact modifiers commonly used for

\footnotetext{
G. Liu · G. Qiu ( $ه)$

Key Laboratory of Rubber-Plastics of Ministry of Education, Shandong Provincial Key Laboratory of Rubber-Plastics, Qingdao University of Science and Technology, Qingdao 266042,

People's Republic of China

e-mail: qiuguixue@qust.edu.cn

G. Liu

e-mail: lgy09@163.com
} 
PP, ethylene-propylene-diene terpolymers (EPDM) are widely used in commercial impact PP resins and considered to be the most effective kind. Although EPDM addition can improve the impact strength of blends, it has some problems. For example, the EPDM are not always in pellet form which decreases the productive effectiveness. In particularly, the viscosity of EPDM is higher than PP which causes dispersion problems and unstable extrusion in processing. So POE has been considered as a substitute for EPDM as a kind of modifier for PP, Da Silva et al. [1]. evaluated the effectiveness of the new polyethylene elastomer (POE) as an impact modifier for PP and compared it with the conventional impact modifier of EPDM. Results indicated that PP/POE and PP/EPDM blends presented similar mechanical performance, but POE showed a better processability. Yang et al. [2] studied the brittle-ductile transition of PP/POE blends in both impact and high speed tensile tests. Results showed that the addition of POE decreased the shear deformation greatly because of the elastomer particle voids and the brittle-ductile transition (BDT) in both impact and high speed tensile tests shared the same mechanism.

In this study, the polyolefin elastomer (POE, ethylene-octene copolymer) was used as an impact modifier for PP to produce automobile bumpers. In addition, small amounts of HDPE were added to the PP in order to improve the toughness of binary PP/POE blends. The effect of the morphology of the composites on mechanical properties of the blends was examined by scanning electron microscopy (SEM).

\section{Experiments}

Materials and blend preparation

The PP used in this study was grade K7708 with a MFI of $10.0 \mathrm{~g} / 10 \mathrm{~min}$ at $230{ }^{\circ} \mathrm{C}$, $2.16 \mathrm{~kg}$. It had some ethylene propylene rubber (20\%, BUNA EP G 2070 P VP, Lanxess Chemistry) added to improve its toughness and was supplied by Beijing Yan Shan Petrochemistry Co. HDPE used was grade DGD6084 with a melt index of $0.8 \mathrm{~g} / 10 \mathrm{~min}$ at $190{ }^{\circ} \mathrm{C}, 5.00 \mathrm{~kg}$, produced by Qilu Petrochemistry Co., The POE used was grade Engage 8150 manufactured by Du Pont/Dow Elastomers, with an octene content of $39 \%$ and a melt index of $0.5 \mathrm{~g} / 10 \mathrm{~min}$ at $190{ }^{\circ} \mathrm{C}, 5.00 \mathrm{~kg}$.

PP, POE, and HDPE of varied ratios were extruded with a single-screw mixer (length to diameter ratio equal to 25/1). The temperature of the mixing section was set at $170-210^{\circ} \mathrm{C}$, and the screw rate was set at $45 \mathrm{rpm}$. Basically, the sample preparation consisted of five processes, extrusion, cooling, drying, granulating, and injection molding. Injection molding pressure was $120 \mathrm{MPa}$, injection temperature was $200{ }^{\circ} \mathrm{C}$ and shot cycle was $1 \mathrm{~min}$.

\section{Measurements}

The melt flow rate (MFR) of virgin polymers and PP blends were determined at the temperature of $230{ }^{\circ} \mathrm{C}$ and the load of $2.16 \mathrm{~kg}$ using a melt index (model $\mu$ PXRZ- 
400C) manufactured by Kejiao Instrument Factory of Jilin University, according to GB3682-83.

The tensile test was conducted at room temperature using tensile machine (model LJ-1000) supplied by Guangzhou Instrument Co., China according to GB/T1042-92, at a tensile rate of $50 \mathrm{~mm} / \mathrm{min}$. The bending test was measured according to GB1042-79 at a strain rate of $25 \mathrm{~mm} / \mathrm{min}$. Izod impact strength was tested according to GB1843-96 (U-notched) on an Impact Tester (model XCT-40) provided by Hebei Chengde Material Testing Machine Co., China. All results aforementioned were the average of at least five measurements.

The morphology of the blends were examined via SEM, model JSM-840 manufactured by JEOL Co., Japan. Fracture surfaces of notched Izod specimens were studied by SEM at room temperature in order to study the rupture mechanism. At the same time, in order to determine the particle size and the distribution of the dispersed impact modifier, chemically etched fracture surfaces were imaged. Etching for 4 days with xylene selectively dissolved the POE from the fracture surfaces and left the PP undissolved. The surfaces were washed with xylene to avoid the reprecipitation. All SEM samples were gold coated prior to SEM observation.

DSC (DSC204, manufactured by Netzsch Co., Germany) was used to determine the effect of POE content on the crystallinity of PP. In the test, after the samples of $5 \mathrm{mg}$ were placed in the testing chamber, the temperature was raised from 30 to $200{ }^{\circ} \mathrm{C}$ and then kept at $200{ }^{\circ} \mathrm{C}$ for 5 min to eliminate the thermal history. After that, the temperature was decreased to $30{ }^{\circ} \mathrm{C}$ for $5 \mathrm{~min}$ then raised to $200{ }^{\circ} \mathrm{C}$ both with a rate of $10{ }^{\circ} \mathrm{C} / \mathrm{min}$. First cooling and second heating curves of crystallization and melting were recorded.

\section{Results and discussion}

The choice of materials

POE is a new thermoplastic elastomer that has many advantages, such as excellent toughness, good processability, good compatibility with other polyolefins (PO), and high melt strength. Metallocene catalysts for olefin polymerization have allowed the control of molecular weight distribution and the proportion and distribution of comonomer incorporation [3]. Moreover, the toughness of POE increases with the increase of the octene concentration. So, we choose the POE grade Engage 8150 whose content of octene was $39 \%$, as the impact modifier to toughen PP. The studied range of POE concentration in this paper was 0-20\%.

The effect of POE concentration on properties of the blends

MFR is a measure of the flowability of polymers. As illustrated in Fig. 1, the flowability of the blends decreased with the increase of POE.

This was attributed to the low MFR of PP itself and the increased entanglement among molecular chains because of the addition of POE with regular short and long side chains which affect the relative mobility of the molecules. On the contrary, impact toughness increased with the increase of POE. The results can be viewed as 


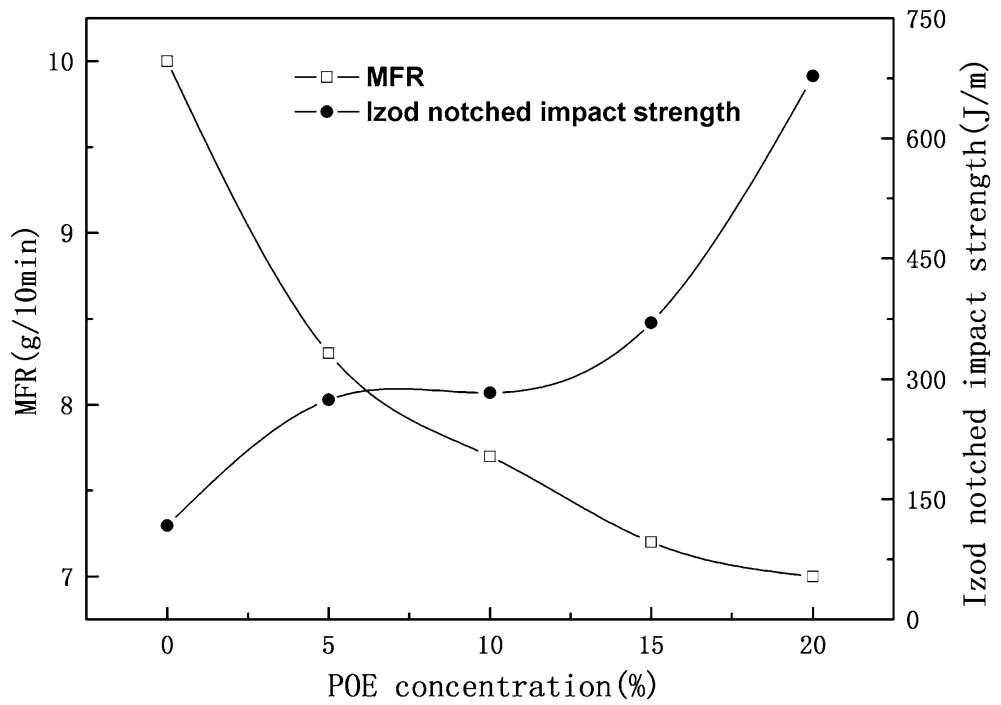

Fig. 1 The effect of POE concentration on MFR and impact strength of the blends

due to the lowering of the crystallinity of the blends in relation to pure PP which will be discussed later. Furthermore, the increase of toughness was small in the range of POE concentration of 5-15\%. When the content of POE reached $20 \%$ in the blends, a tough-brittle transition occurred and the toughness of the blends sharply increased. The results showed that the POE had a significant toughening effect on the PP copolymer. Paul and Kale [4] also found that the tough-brittle transition occurred at the 20-25\% of POE concentration in their studied system.

Table 1 showed that the addition of POE to the PP matrix resulted in a decrease of yield strength and bending strength, and an increase of breaking elongation. These results can also be discussed below the lowering of the crystallinity of the blends in relation to pure PP.

The effect of HDPE concentration on mechanical property of blends

HDPE was added into the blends for a fixed POE content of $15 \%$ in order to improve toughness and decrease the cost of the blends. HDPE, by itself, is

Table 1 The effect of POE concentration on mechanical properties of the blends

\begin{tabular}{lllll}
\hline $\begin{array}{l}\text { PP copolymer } \\
(\mathrm{wt} \%)\end{array}$ & $\begin{array}{l}\text { POE } \\
(\mathrm{wt} \%)\end{array}$ & $\begin{array}{l}\text { Yield strength } \\
(\mathrm{MPa})\end{array}$ & $\begin{array}{l}\text { Elongation at } \\
\text { break }(\%)\end{array}$ & $\begin{array}{l}\text { Bending strength } \\
(\mathrm{MPa})\end{array}$ \\
\hline 100 & 0 & 26.2 & 135 & 36.7 \\
95 & 5 & 22.4 & 270 & 28.9 \\
90 & 10 & 22.0 & 317 & 28.1 \\
85 & 15 & 21.1 & 328 & 27.4 \\
80 & 20 & 19.6 & $>500$ & 24.8 \\
\hline
\end{tabular}


ineffective in toughing PP, presumably because PP and HDPE are both crystalline polymers and they tend to keep their own crystal behavior although some entanglement takes place in the amorphous part. The HDPE can not initiate crazes nor arrest crack growth as effectively as rubber particles. This behavior may be caused by poor PP-HDPE adhesion as well as by the non-rubberlike characteristics of HDPE [5]. However, as illustrated in Table 2, HDPE used in admixture with POE was an effective toughening agent for PP. In the PP-rich PP/POE blend, $1 \%$ HDPE significantly improved the impact strength and breaking elongation of the blends but decreased its yield and bending strength. Thus, the use of HDPE had an obvious synergistic effect on toughening. However, further increasing the HDPE content caused additional slight declines in yield strength and bending strength. This may be because POE acted as ethylene-propylene rubber.

Our results showed that the proper content of HDPE was 1-3\% in the blends at the $15 \%$ POE level. Bartlett et al. [6] have claimed that the appropriate amount of ethylene-propylene rubber could greatly improve the ductility of PP/HDPE but with a corresponding decrease in strength and modulus.

\section{Morphology and properties of binary PP-POE blends}

It is well known that a close interrelation exists between physico-mechanical properties and phase structure of blends. The physico-mechanical properties of blends have been shown to depend on the average particle size, particle-size distribution, and some molecular features (such as average molecular mass, degree of crosslinking, etc.) of the elastomeric impact modifier forming the dispersed phase in the blends, in addition to the composition and processing conditions.

In this study, to investigate the fracture mechanism of the blends, impact fracture surfaces (not etched) were directly observed by SEM. The brittle-ductile transition of the blends could be seen from the morphology of the surfaces.

Figure 2 shows representative SEM micrographs of impact fracture surfaces of injection-molded specimens of the PP/POE blends. In Fig. 2a, the globular particles represent the EPR component. The voids in the micrograph were produced because of falling out of EPR particles. In Fig. 2b, c, the fracture surfaces became rougher and the POE domains were dispersed in the matrix evenly. The increase of POE content from 5 to $15 \%$ made no obvious difference in SEM micrographs. So, we can say that 5-15\% POE could improve the toughness of the blends, but the

Table 2 The effect of HDPE concentration on mechanical properties of the blend containing $15 \%$ POE

\begin{tabular}{lllllll}
\hline $\begin{array}{l}\text { PP } \\
\text { copolymer } \\
(\mathrm{wt} \%)\end{array}$ & $\begin{array}{l}\text { HDPE } \\
(\mathrm{wt} \%)\end{array}$ & $\begin{array}{l}\text { MFR } \\
(\mathrm{g} / 10 \mathrm{~min})\end{array}$ & $\begin{array}{l}\text { Yield } \\
\text { strength } \\
(\mathrm{MPa})\end{array}$ & $\begin{array}{l}\text { Elongation at } \\
\text { break }(\%)\end{array}$ & $\begin{array}{l}\text { Bending } \\
\text { strength } \\
(\mathrm{MPa})\end{array}$ & $\begin{array}{l}\text { Izod impact } \\
\text { strength } \\
(\mathrm{J} / \mathrm{m})\end{array}$ \\
\hline 85 & 0 & 7.3 & 21.1 & 328 & 27.4 & 370 \\
84 & 1 & 7.2 & 20.7 & $>500$ & 24.5 & 513 \\
82 & 3 & 6.5 & 19.8 & $>500$ & 23.9 & 547 \\
80 & 5 & 6.1 & 19.3 & $>500$ & 23.1 & 573 \\
\hline
\end{tabular}


increase was limited. In Fig. 2d, the highest POE content $(20 \%)$, there was a cocontinuous phase structure, rough surface which are the typical characterizations of tough fracture.

Etching with xylene at room temperature for 3-4 days selectively dissolved the POE and some amorphous parts of PP from the fracture surfaces which were easily etched then washed with xylene to avoid the reprecipitation. Figure 3 shows voids in the PP matrix. The hollow zones represent the locations of the solvent-etched POE and amorphous PP in the PP/POE blends. With the increase of POE content, the size of the dispersed POE phase increased and the morphology of the disperse phase particles changed.

In Fig. 3a, the size of the dispersed phase particles was smaller than in the others. Moreover, it presented a smooth fracture surface than the other samples because the interface of the two phases is clear and no deformation was observed. In Fig. 3b, the size of the dispersed particles became larger and were distributed in the PP matrix with morphology of slender and atactic. Figure $3 \mathrm{c}$ showed the fracture surfaces when the POE concentration was $20 \%$, and we can see clearly that some of the PP matrix has a lamellar morphology. The phenomena could be explained by a tight
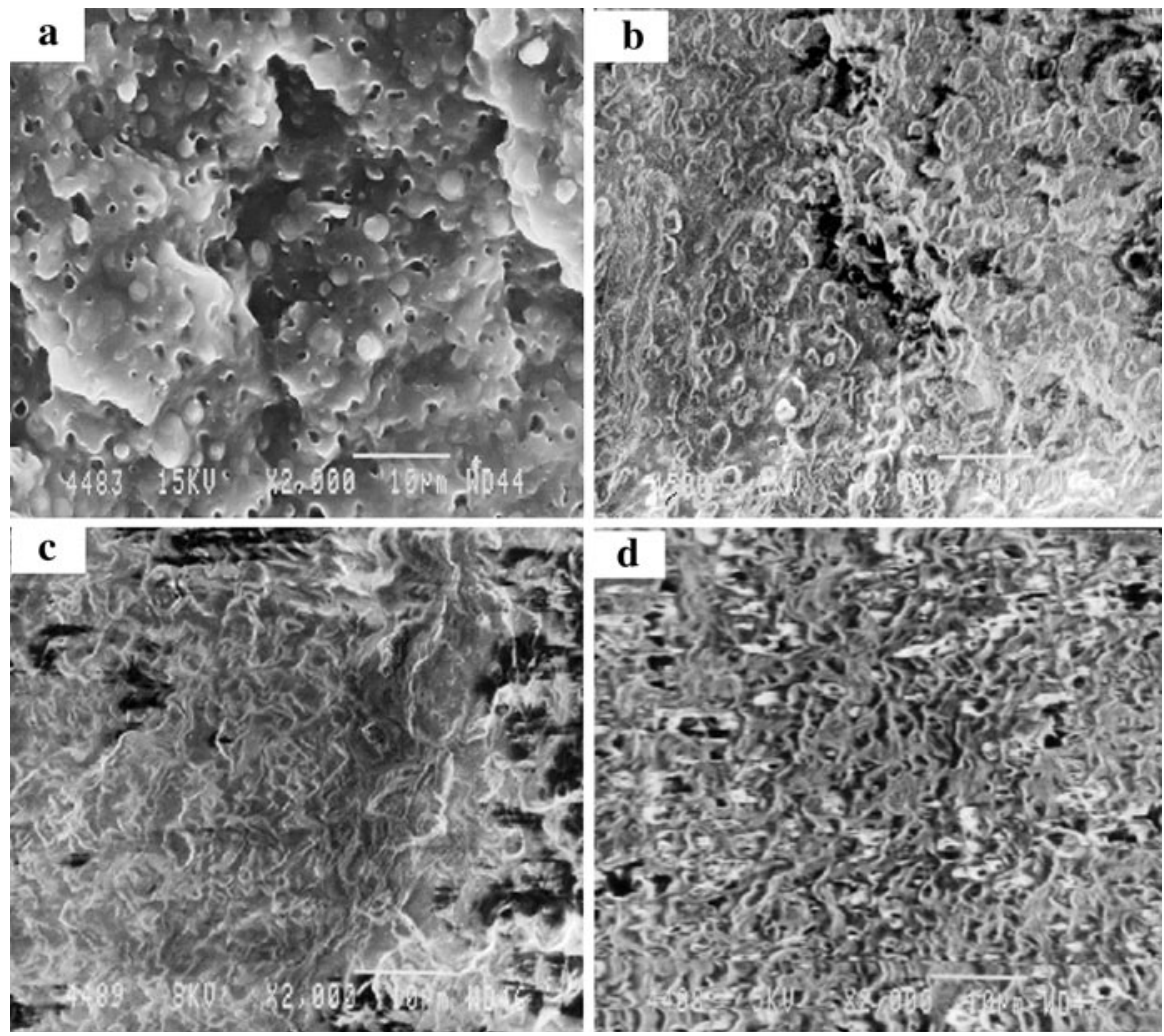

Fig. 2 SEM micrographs of impact fracture surfaces of PP based blends containing various amount of POE a $0 \%$ of POE, b $5 \%$ OF POE, c $15 \%$ of POE, d $20 \%$ of POE 

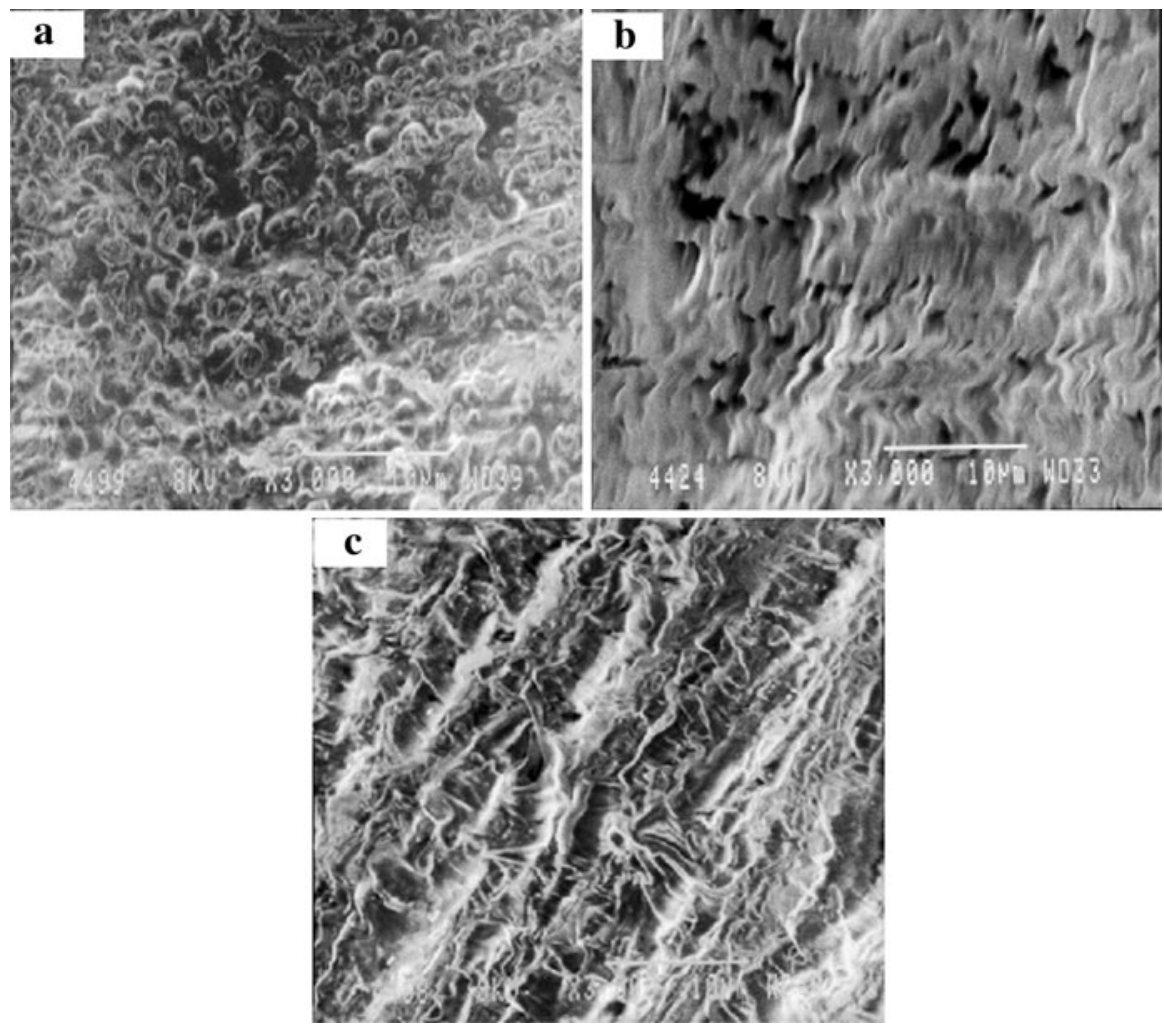

Fig. 3 SEM micrographs of xylene etched fracture surfaces of PP/POE blends a $5 \%$ of POE, b $15 \%$ of POE, c $20 \%$ of POE

interface adhesion of PP and POE caused by the deformation of PP when the blends were impactive fractured. These phenomena could also be viewed as the results of the good compatibility between PP and POE, and increase of POE content can make the shear viscosity of the whole system higher and make the dispersed phase finer.

In view of the images, we can say that dispersed POE initiates crazes and shear band in blends as stress concentration factors. At the same time, interference of great quantities of crazes decreased the stress at the edges of the crazes, preventing further development of crazes and finally improved the toughness of materials.

DSC analysis of the PP/POE blends with different POE content

DSC was used in this study to analyze the effect of POE content on the crystallinity behavior of PP. The relative data are shown in Table 3 . The crystallinity $X_{\mathrm{c}}(\%)$ can be calculated by Eq. 1, as follows, with the results shown in Table 3.

$$
\text { Crystallinity } X_{\mathrm{c}}(\%)=\Delta H_{\mathrm{m}} /\left(\Delta H_{\mathrm{ma}} \cdot w\right)
$$

where $X_{\mathrm{c}}$ is the crystallinity degree of the PP/POE blends, $\Delta H_{\mathrm{m}}$ is the melting enthalpy of the PP/POE blends, $\Delta H_{\mathrm{ma}}$ is $209 \mathrm{~J} / \mathrm{g}$ [7] which is the standard enthalpy 
Table 3 DSC data of the PP/POE blends

\begin{tabular}{lcccc}
\hline Sample of PP/POE & $100 / 0$ & $95 / 5$ & $85 / 15$ & $80 / 20$ \\
\hline$T_{\mathrm{m}} /{ }^{\circ} \mathrm{C}$ & 166.3 & 165.2 & 165.1 & 164.4 \\
$\Delta H_{\mathrm{m}} / \mathrm{J} \mathrm{g}^{-1}$ & 93.33 & 75.85 & 69.59 & 60.17 \\
$\Delta H_{\mathrm{c}} / \mathrm{J} \mathrm{g}$ & 97.12 & 80.62 & 78.07 & 74.81 \\
$X_{\mathrm{c}} / \%$ & 44.66 & 38.20 & 39.17 & 35.99 \\
\hline
\end{tabular}

$T_{\mathrm{m}}$ is the temperature of the melting peaks of the PP/POE blends, $\Delta H_{\mathrm{m}}$ is the melting enthalpy of the PP/ POE blends and not normalized for the POE content, $\Delta H_{\mathrm{c}}$ is the crystalline enthalpy of the PP/POE blends, $X_{\mathrm{c}}$ is the crystallinity degree of the PP/POE blends based on $\Delta H_{\mathrm{m}}$

of PP when it is $100 \%$ crystalline, and $w$ is the weight percent of PP in the PP/POE blends.

As shown in Table 3, there was only one melting peak in the curves and the $T_{\mathrm{ms}}$ decreased with the increase of POE content. This was expected because of the lower $T_{\mathrm{m}}$ of POE (about $55^{\circ} \mathrm{C}$ ). Both $\Delta H_{\mathrm{m}}$ and $\Delta H_{\mathrm{c}}$ declined with the rise of POE amount, which indicates that the addition of POE affected the crystallinity of PP. $X_{\mathrm{c}}$ decreased with increasing POE. This behavior can help to explain the toughing effectiveness of POE on PP, as described above.

\section{Conclusions}

POE significantly improved the impact strength and breaking elongation in PP/POE blends, but resulted in a decrease of MFR, yield strength and bending strength. The most useful content of POE was 15-20\%. The addition of a small amount of HDPE had an obvious synergistic effect on toughening. 1\% HDPE can significantly improve the impact strength and breaking elongation of the blends. Further increasing the HDPE content caused a mild decrease in yield strength and bending strength. The most useful content of HDPE was 1-3\%. SEM showed that the impact fracture surface of composites of PP/POE had a ductile appearance at $20 \%$ POE content due to partial compatibility. DSC analysis indicated that the crystallinity of PP decreased with the increase of POE content. We suggest this caused the improved toughness of the PP blends.

Acknowledgments The authors are grateful to all the suppliers of raw materials used in this study.

Open Access This article is distributed under the terms of the Creative Commons Attribution License which permits any use, distribution, and reproduction in any medium, provided the original author(s) and the source are credited.

\section{References}

1. Da Silva ALN, Tavares MIB, Politano DP, Coutinho FMB, Rocha MCG (1997) Polymer blends based on polyolefin elastomer and polypropylene. J Appl Polym Sci 66(10):2005-2014 
2. Yang J, Zhang Y, Zhang Y (2003) Brittle-ductile transition of PP/POE blends in both impact and high speed tensile tests. Polymer 44:5047

3. Zhao J et al (2009) The thermodynamic behavior and morphology of PP/POE blends prepared by meltand solution-mixing methods. J Mater Sci 44: 2171

4. Paul S, Kale DD (2000) Impact modification of polypropylene copolymer with a polyolefinic elastomer. J Appl Polym Sci 76:1480

5. Stehling FC, Huff T, Speed CS, Wissler G (1981) Structure and properties of rubber-modified polypropylene impact blends. J Appl Polym Sci 26:2693

6. Bartlett DW, Barlow JW, Paul DR (1982) Mechanical properties of blends containing HDPE and PP. J Appl Polym Sci 27:2351

7. Svoboda P, Zeng C, Wang H, Lee LJ, Tomasko DL (2002) Morphology and mechanical properties of polypropylene/organoclay nanocomposites. J Appl Polym Sci 85:1562 